\title{
Impact of smoking on neuroactive steroids
}

\section{Hana Hruškovičová, Michaela Dušková, Kateřina Šimůnková, Hana Pospíšilová, Martin Hill, Karel Vondra, Luboslav Stárka}

\section{Introduction:}

\section{Institute of Endocrinology, Prague}

Cigarette smoking still presents one of the most widespread and persistent addictions. The dependence on tobacco, diagnosis

$\mathrm{F} \mathrm{17,} \mathrm{is} \mathrm{an} \mathrm{independent} \mathrm{condition} \mathrm{in} \mathrm{the} \mathrm{International} \mathrm{classification} \mathrm{of} \mathrm{diseases.} \mathrm{Chronic} \mathrm{smoking} \mathrm{can} \mathrm{cause} \mathrm{impairment} \mathrm{of} \mathrm{fertility} \mathrm{in} \mathrm{both}$ sexes. The male reproductive system is more resilient; mainly a negative shift in spermiogram parameters has been described in male smokers. However, only a small number of studies were dedicated to influence of smoking on levels of steroid hormones and their neuroactive metabolites. It is generally accepted that neuroactive steroids influence effects of tobacco, and also the level of dependency on tobacco. In our study we monitored levels of neuroactive steroids in men smokers during one year of smoking abstinence and we looked for a possible predictive marker of success in smoking cessation.

Methods: We examined 76 men before initiating of smoking cessation, after 6 weeks and after a year of abstinence. According to success we divided our samples to 3 groups, 26 men were successful in smoking cessation (group S+), 16 men stopped smoking only for 6 weeks (group $\mathrm{S} \pm$ ) and 34 men did not stop smoking at all (S-). We measured basic anthropometric data, levels of steroids hormones and their neuroactive metabolites by GC-MS.

The local Ethics Committee approved the study and all patients signed an informed consent form before taking part in the study.

\section{Results:}

In the baseline hormonal data we found lowel levels of testosterone and some other neuroactive metabolites of androgens in men, who failed smoking cessation ( $\mathrm{S}-$ and $\mathrm{S} \pm$ ) compared to men succesfull in smoking cessation (S+). Moreover the lower the levels, the higher the probability of failure.
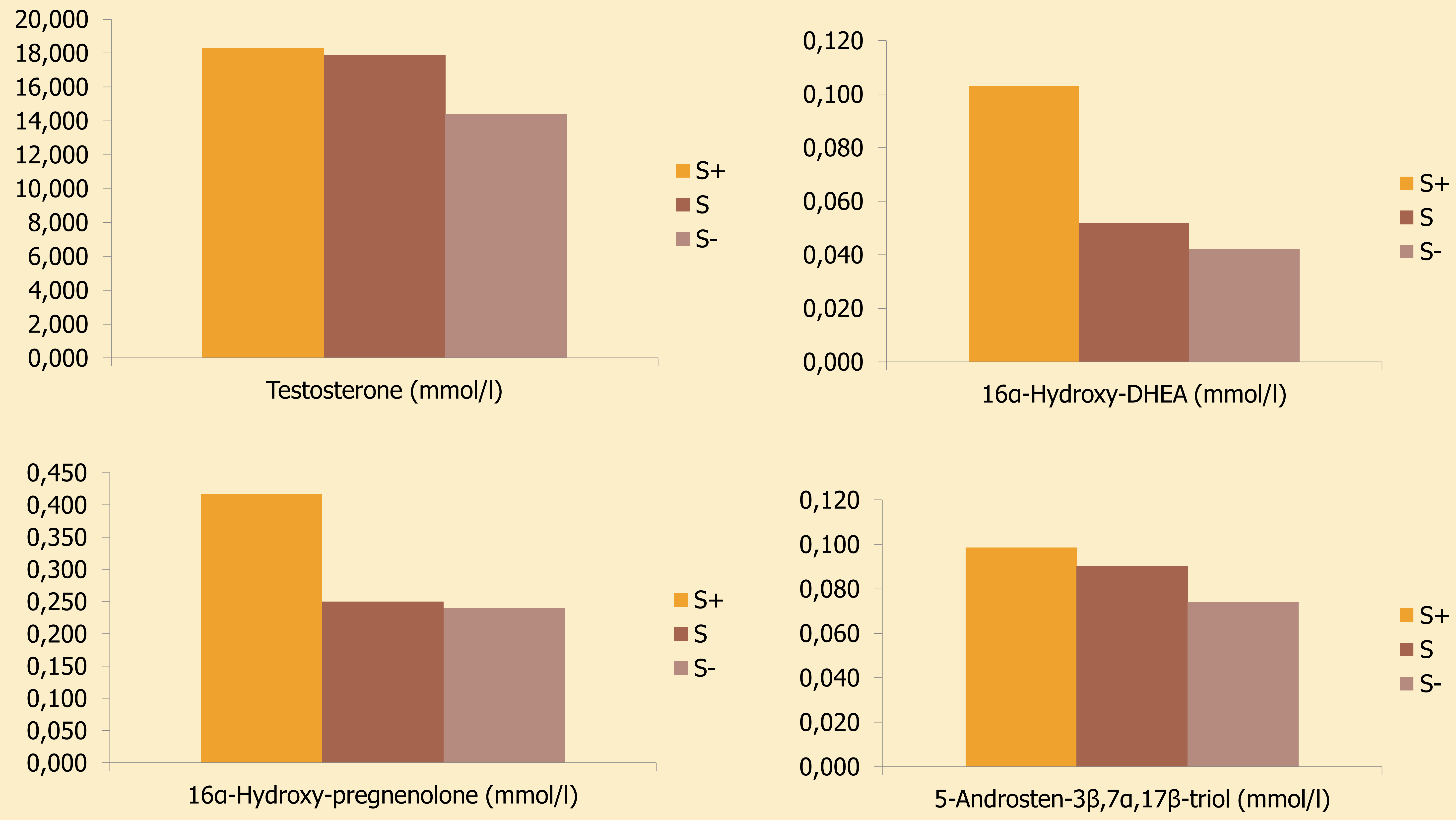

\section{Conclusion:}

The lower levels of testosterone and some neuroactive metabolites of androgens in men were associated with failure in smoking cessation. Moreover the lower the levels of androgens, the less probable success in smoking cessation. 